\title{
Observation of Charged Particles Aboard the Scientific Satellite JIKIKEN (EXOS-B)
}

\author{
Haruya Kubo, Toshifumi MukaI, and Nobuki Kawashima \\ Institute of Space and Aeronautical Science, University of Tokyo, \\ Komaba, Meguro-ku, Tokyo, Japan
}

(Received October 27, 1980)

\begin{abstract}
Charged particle detectors (ESP) on board the Japanese sixth scientific satellite JIKIKEN have measured electron and ion fluxes in the energy ranges of a few $\mathrm{eV}$ up to $10 \mathrm{keV}$ and of $10 \mathrm{eV}$ up to $30 \mathrm{keV}$, respectively, in the magnetosphere of $L$-shells from $L \cong 2.5$ to $L \cong 8$. Main results are: (1) On quiet days, significant fluxes of electrons and ions were rarely observed. (2) On disturbed days, intense fluxes of $\mathrm{keV}$-electrons were observed at $L \geqq 4$, suggesting a plasma injection from the plasma sheet. Ring current ions in the energy range above $1 \mathrm{keV}$ were also observed on disturbed days after magnetic storm.
\end{abstract}

\section{Introduction}

The Japanese sixth scientific satellite EXOS-B (JIKIKEN) was launched by M-3H-3 rocket at 1400 JST (0500 UT) on September 16, 1978 from Kagoshima Space Center (Uchinoura) into an elliptic orbit of inclination $31^{\circ}$, the initial apogee and perigee being $30,055 \mathrm{~km}$ and $227 \mathrm{~km}$, respectively. JIKIKEN explores the inner magnetosphere of middle and low magnetic latitude regions and particle measurements by electron and ion energy analyzers (ESP $(E)$ and ESP $(I)$ ) on board which will shed some new light on the magnetospheric physics.

The purposes of the charged particle measurements on board JIKIKEN are as follows:

1) To investigate the dynamics of electrons and ions in the inner magnetosphere.

2) To investigate wave-particle interaction in the inner magnetosphere together with the wave measurements.

3) To observe a response of magnetospheric plasma when an active experiment, that is SPW (high power radio wave emission) and/or CBE (controlled electron beam emission) is performed.

Charged particle measurements in the inner magnetosphere have begun since Rev. 65 (October 9, 1978) when the high voltage power supplies of charged praticle detectors (ESP) were first switched on. The data reported here were obtained 
during about 6 months, that is, from October 9, 1978 (Rev. 65) to April 25, 1979 (Rev. 628). During this period of observation the satellite surveyed the magnetosphere of $L$-shells between $L \cong 2.5$ and $L \cong 8$ in the MLT sectors of midnight to dusk and late afternoon.

In this paper, only the No. 1 item in the above purposes is described. In section 2 instrumentation is described and in section 3, the data are presented and discussions are given.

\section{Instrumentation}

Charged particle detectors (ESP) are composed of an electron sensor, an ion sensor and electronic circuits. The electron and ion sensors measure electrons and ions in the energy ranges from a few $\mathrm{eV}$ to $10 \mathrm{keV}$ and from $10 \mathrm{eV}$ to $30 \mathrm{keV}$, respectively. Characteristics of the sensors are summarized in Table 1.

ESP installed in the satellite is shown in Fig. 1. It is composed of charged particle energy analyzers, a sweep voltage power supply, channeltrons, high voltage power supplies for channeltrons and pre-amplifiers. A better understanding of the structure of the electron sensor is obtained by a schematic drawing in Fig. 2 which is a vertical cross section of Fig. 1. The electron sensor is composed of a front collimator, a hemispherical electronstatic energy analyzer with inner and outer radii of $28 \mathrm{~mm}$ and $32 \mathrm{~mm}$, respectively, and a channeltron as a detector. The ion sensor is composed of a collinator, a $60^{\circ}$-cylindrical electrostatic analyzer with inner and outer radii of $58.5 \mathrm{~mm}$ and $61.5 \mathrm{~mm}$, respectively, and a channeltron. Optical axes of both electron and ion collimators are parallel and installed in the

Table 1. Characteristics of the sensors.

\begin{tabular}{|c|c|c|}
\hline & \multicolumn{2}{|c|}{ Sensors } \\
\hline & Electron & Ion \\
\hline Type & Hemispherical & Cylindrical $\left(60^{\circ}\right.$ Sector $)$ \\
\hline Energy range & a few eV-10 keV & $10 \mathrm{eV}-30 \mathrm{keV}$ \\
\hline Dynamic range & $\begin{array}{l}10^{4}-10^{9} \text { electrons/ } \\
\left.\mathrm{cm}^{2} \text { sec str eV (at } 100 \mathrm{eV}\right)\end{array}$ & $\begin{array}{l}10^{4}-10^{9} \text { ions/ } \\
\left.\mathrm{cm}^{2} \mathrm{sec} \text { str eV (at } 100 \mathrm{eV}\right)\end{array}$ \\
\hline \multicolumn{3}{|l|}{ Energy resolution } \\
\hline$\Delta E / E$ & $3 \% *$ & $5 \% *$ \\
\hline Geometrical factor & $\begin{array}{l}1.27 \times 10^{-3} \mathrm{~cm}^{2} \mathrm{str} \\
\text { experimental }\end{array}$ & $\begin{array}{l}1.0 \times 10^{-3} \mathrm{~cm}^{2} \mathrm{str} \\
\text { calcualtion }\end{array}$ \\
\hline $\begin{array}{l}\text { Sensitivity of } \\
\text { channeltron }\end{array}$ & $\sim 0.5$ & $\sim 1.0$ \\
\hline $\begin{array}{l}\text { Direction of the } \\
\text { collimator }\end{array}$ & $\begin{array}{l}\text { Perpendicular to the spin } \\
\text { axis of the satellite }\end{array}$ & The samt to the left \\
\hline Field of view & $4^{\circ} \times 13^{\circ}$ & $3^{\circ} \times 3^{\circ}$ \\
\hline
\end{tabular}

* These energy resolutions are those of sensors only. Concerning the energy width of the data refer to Table 2 . 


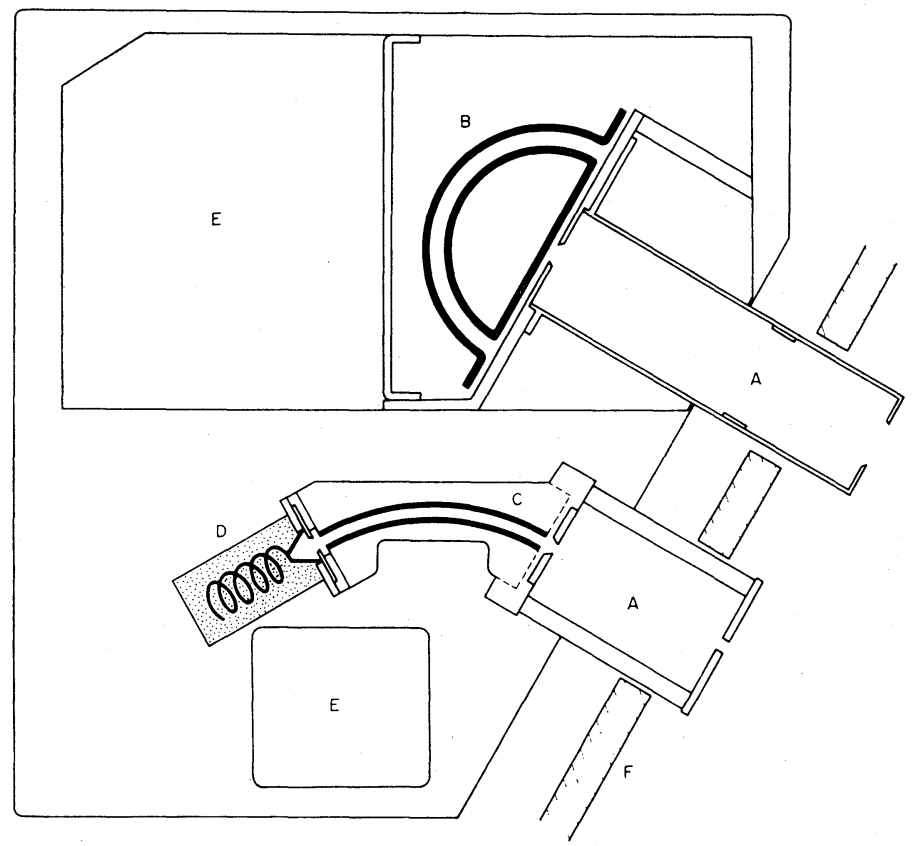

Fig. 1. Schematic diagram of charged particle detectors. A: Collimators. B: Hemispherical electrostatic analyzer (for electrons). C: Cylindrical electrostatic analyzer (for ions). D: Channeltron and its support (for electron sensor see Fig. 2). E: Sweep voltage power supply, high voltage power supplies for channeltrons, charge amplifiers, and pulse discriminating and shaping circuits. F: Surface of the satellite.

satellite so that they are perpendicular to the satellite main axis of inertia. Both collimators extrude from the sattellite surface by about $15 \mathrm{~mm}$. Charged particles entering the analyzers through the collimators are deflected by the electic field ( $E \propto 1 / r^{2}$ for the electon analyzer, $E \propto 1 / r$ for the ion analyzer), and particles having the appropriate energies can pass through the analyzers and impinge onto the channeltrons.

A high voltage of about $3.0 \sim 4.0 \mathrm{kV}$ is applied to the output terminal of the electron detecting channeltron, and accelerating voltage of about $300 \mathrm{~V}$ is applied to the input terminal in order to maintain the counting efficiency nearly constant over the measured energy range (PASCHMANN et al., 1970). For the ion detecting channeltron, a high voltage of $-2.8 \sim-4.0 \mathrm{kV}$ is applied to the input terminal making the detection efficiency nearly constant, and the output terminal is set nearly to the ground potential. These high voltages for channeltrons can be varied by three steps by commands. After the first high voltage switch-on (Rev. 65), high voltages for the electron and ion channeltron were set to the lowest levels (electron: $+2.98 \mathrm{kV}$, ion: $-2.78 \mathrm{kV}$ ). Afterwards on Rev. 378 (January 28, 1979) high voltage for the 


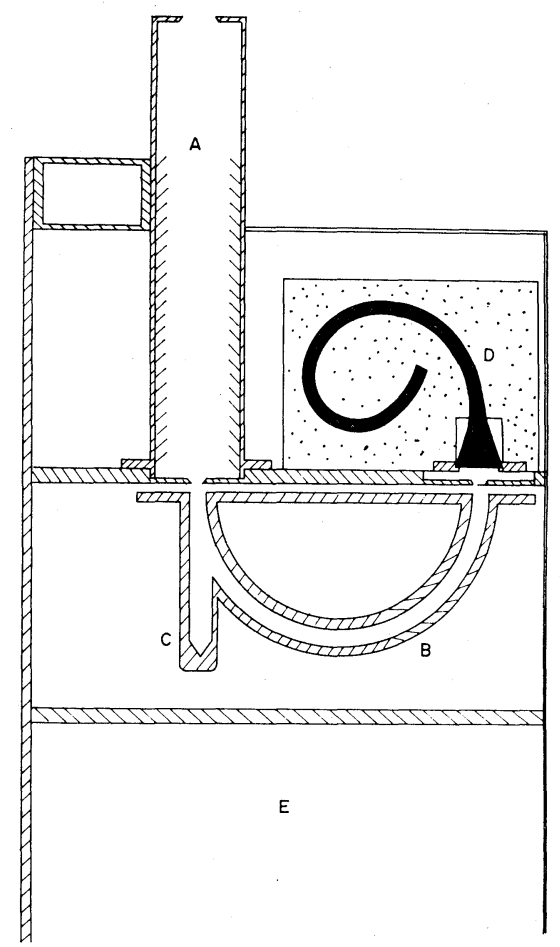

Fig. 2. Schematic diagram of the electron sensor; vertical cross section of Fig. 1. A: Collimator. B: Hemispherical electrostatic analyzer. C: Trap for high energy particles and solar radiation. D: Channeltron and its support. E: Sweep voltage power supply, high voltage power supplies for channeltrons, charge amplifiers, and pulse discriminating and shaping circuits.

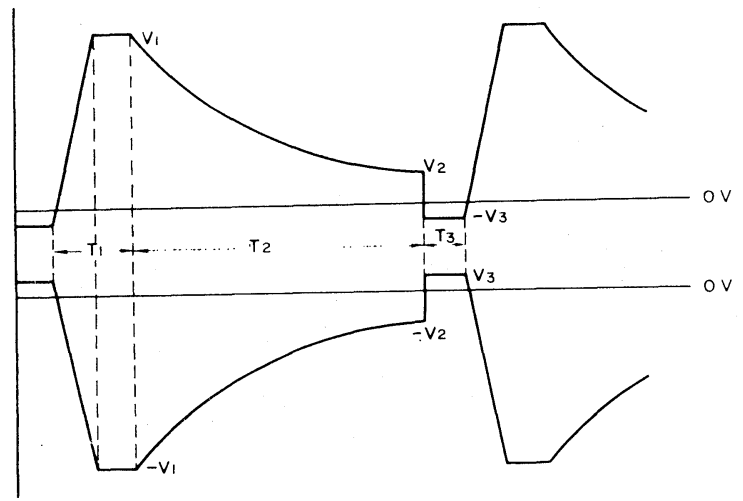

$$
\begin{aligned}
v_{1} & =1500 \mathrm{~V} \\
v_{2} & =0.5 \mathrm{~V} \\
V_{3} & =5 \mathrm{~V} \\
T_{1} & =125 \mathrm{~m} \mathrm{sec} \\
T_{2} & =812.5 \mathrm{~m} \mathrm{sec} \\
T_{3} & =62.5 \mathrm{~m} \mathrm{sec}(1024 \mathrm{bits} / \mathrm{sec}) \\
& =3062.5 \mathrm{~m} \mathrm{sec}(256 \mathrm{bits} / \mathrm{sec})
\end{aligned}
$$

Fig. 3. Waveform of the sweep voltage. 
ion channeltron was altered to the middle level $(-3.34 \mathrm{kV})$.

The analyzer energy is swept simultaneously for electrons and ions, by applying exponentially decaying voltages as shown in Fig. 3, and the repetition frequency is $1 \mathrm{~Hz}$ (when $1024 \mathrm{bits} / \mathrm{sec}$ ) or $0.25 \mathrm{~Hz}$ (when $256 \mathrm{bits} / \mathrm{sec}$ ). A block diagram of the sensors and the count data processing circuits of ESP is shown in Fig. 4. Output charge pulses from both channeltrons are amplified, discriminated, shaped, and are counted continuously every $62.5 \mathrm{msec}$ by 15 -bit counters. These count data are compressed to 8-bit words, stored once in the buffer memories, and then transmitted to the ground by allocated PCM-telemetry words as shown in Fig. 5. Sixteen channels of count data are obtained in one sweep for electrons and ions, respectively. The first two data channels correspond to the rising phase of the sweep voltage waveform. The last one is used for obtaining the background information. As the polarities of the analyzer voltages are reversed at the last data channel, low energy ions pass through the electron analyzer and low energy electrons pass through the ion analyzer, but they are both reflected by the high electric potential barrier at the entrance of channeltron. Thus the last data should account for backgrounds only. However, in reality, these background counts often exceeds the normal electron and ion counts (particularly at the outer radiation belt $L \cong 4 \sim 5$ ). Therefore, it cannot be executed simply to subtract the background counts from the normal counts, and no background correction is done on the data reported in this paper. Then, the meaningful data channels of electrons and ions are 13 out of 16 . The conversion of the compressed 8-bit word of count data to original count can be made by the relation

$$
C=2^{11-S}(D+16),
$$

where $C$ is sample counts (counts $/ 62.5 \mathrm{msec}$ ), and $D$ and $S$ are the decimal value of the upper 4 bits and the lower 4 bits of the 8-bit word, respectively. The differential particle flux $f(E)$, particles $/ \mathrm{cm}^{2}$ sec str $\mathrm{eV}$, is deduced from the observed counts $C$ by the relation

$$
f(E)=C /(G \cdot \Delta E \cdot \tau \cdot \varepsilon),
$$

where $G$ is a geometrical factor in $\left[\mathrm{cm}^{2} \cdot \mathrm{str}\right], \Delta E$ is the energy bandwidth which is proportional to the center energy of measurement, i.e., $\Delta E=R \cdot E$ where $R$ is the energy resolution of the analyzer, $\tau$ is a sampling time which is $62.5 \mathrm{msec}$ in our instrument, and $\varepsilon$ is the detection efficiency. Th : values of $G, R=\Delta E / E$ and $\varepsilon$ are summarized in Table 1. The conversion factor which is defined as $\langle C F\rangle=1 /(G$. $\Delta E \cdot \varepsilon)$ is presented together with the boundary energy and the mean energy in Table 2. We note here that the energy of measurement varies exponentially by a factor of $\sim 1.9$ during the sample time of $62.5 \mathrm{msec}$, and that the mean energy $\sqrt{E_{i} \cdot E_{i+1}}$, where $E_{i}$ is the boundary energy between $(i-1)$-th and $i$-th channels, is used in order to deduce the conversion factor. Temperature dependence of the analyzer voltages is less than $3 \%$. 


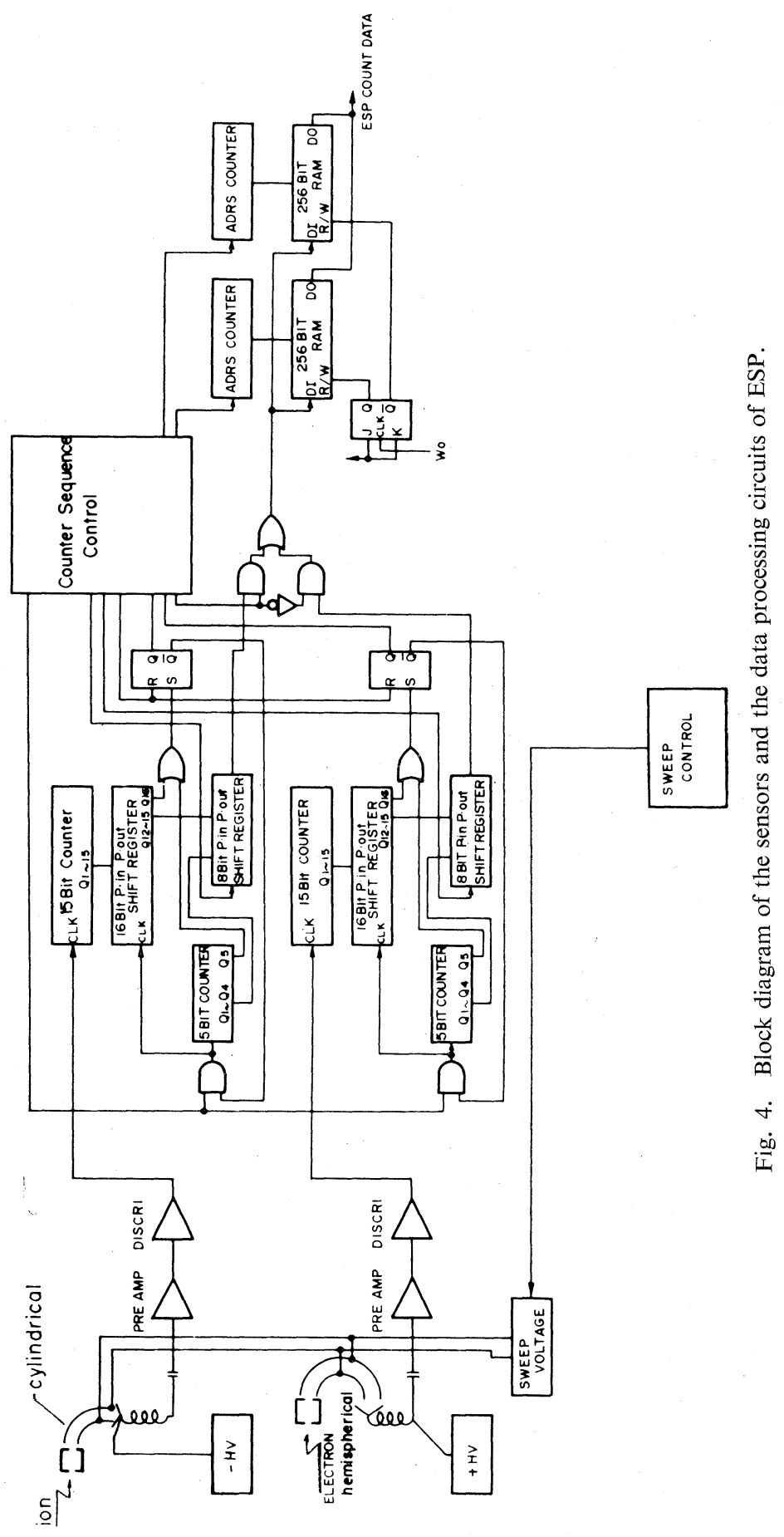




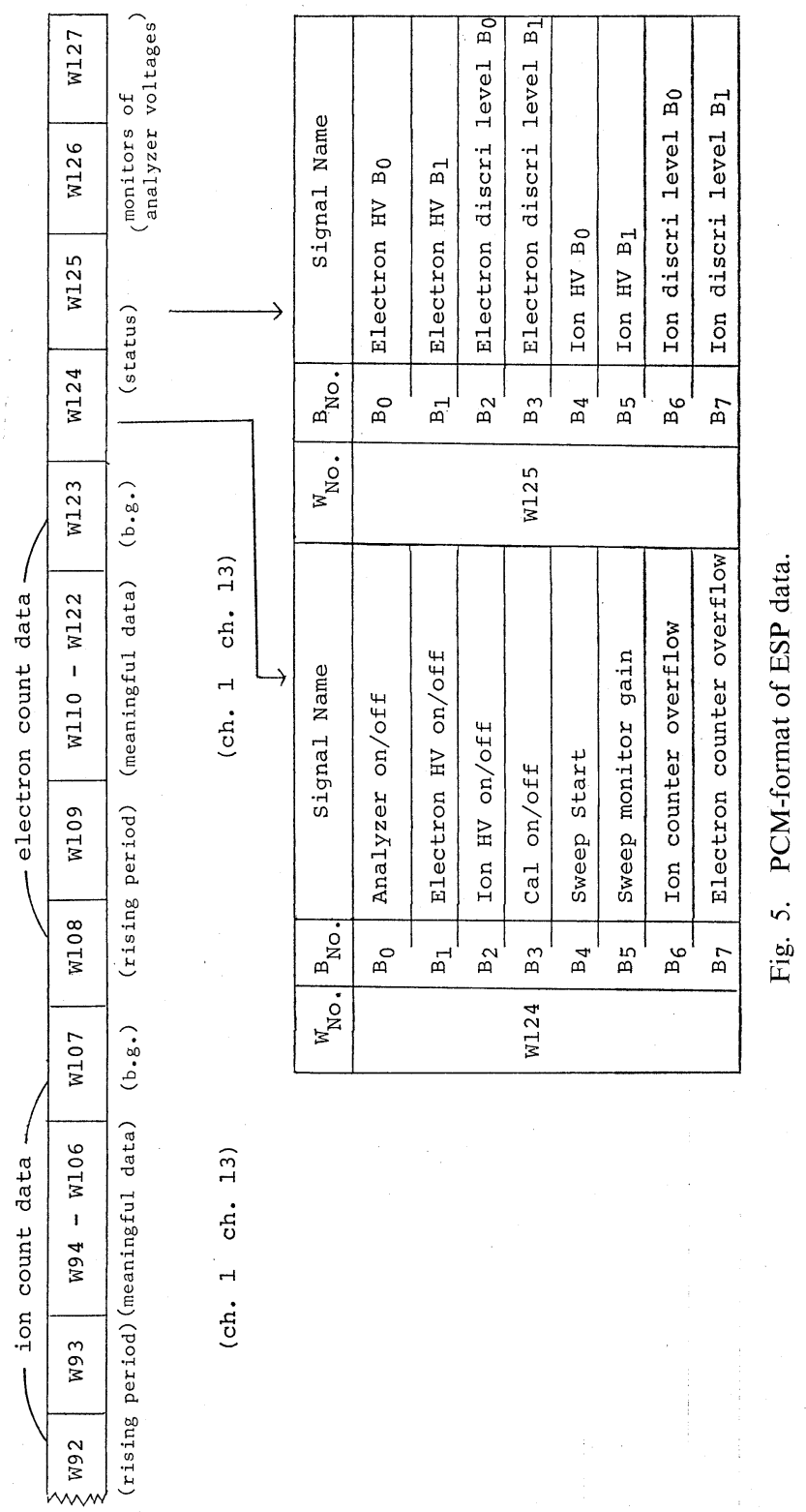




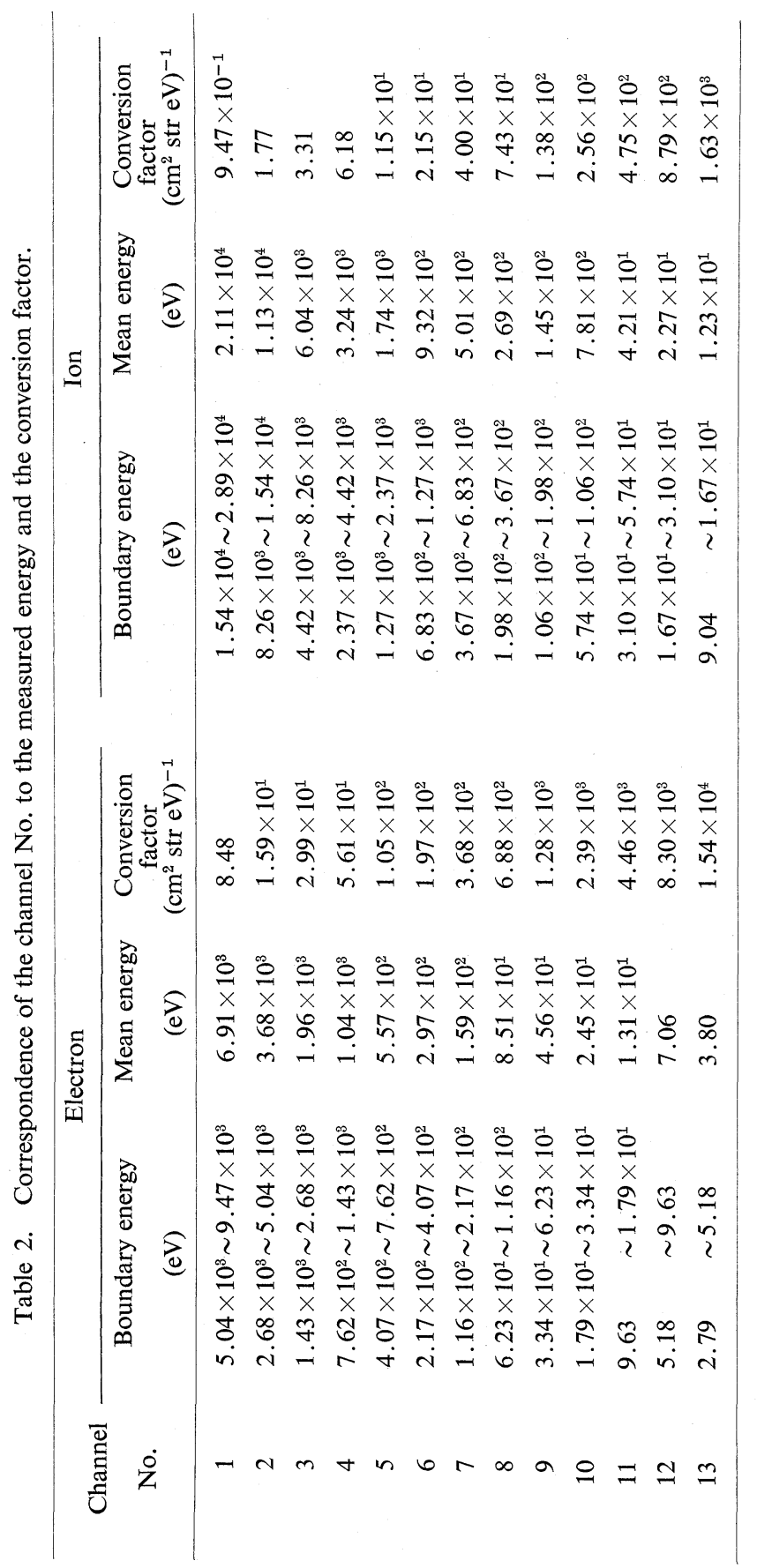


The pitch angel measurement is sensitive to the satellite attitude. Pitch angle distribution can be obtained in the angular range of $0^{\circ}-180^{\circ}$ with the accuracy of $20^{\circ}$ in such a particular case that the geomagnetic field line of force is perpendicular to the spin axis of the satellite. This was the case for $L \gtrsim 5$ in the autumn of 1978 though the satellite attitude had a corning behavior of $\sim 20^{\circ}$ practically. However, unfortunately, malfunction of the magnetometer on board JIKIKEN occurred around the end of November, 1978. Thereupon, the pitch angle must be estimated by the absolute attitude of the satellite deduced from the sun-sensor and the estimated satellite attitude extrapolated from the previous data, using the reference field model, i.e., IGRF. This estimation will be performed, in the near future, with the aid of experimenters of magnetic field (MGF).

\section{Data and Discussions}

The most convenient presentation form of data is a so-called $E$ - $t$ (energytime) diagram. The example of the 20-min data on Rev. 143 (November 6, 1978) is shown in Fig. 6 in which the sun angle (the angle between the orientation of the sensor collimator and the Sun) and the particle pitch angle are also presented. Abscissa scale is time (UT) and the orbital positions of the satellite are presented every $2 \mathrm{~min}$. Ordinate scale in the $E-t$ diagram is particle energy $(\mathrm{eV})$. The respective counts of 13 channels for ions and electrons are shown every $4 \mathrm{sec}$ with gray-shading which is composed of dot-matrix. The shading level which is proportional to $\log _{10} C$ is shown in the upper-right corner in the figure. The counts of background channels are also shown in this figure. If both sides of Eq. (2) are multiplied by $E$, the differential energy flux $E \cdot f(E)$ is obtained by the following relation:

$$
E \cdot f(E)=C /(G \cdot R \cdot \tau \cdot \varepsilon) \propto C .
$$

That is, the counts $C$ is proportional to the differential energy flux. Weighting of the counts inversely proportional to the mean energy yields a differential number flux, while weighting directly proportional to the mean energy gives the total energy carried in each channel of the spectrum.

At first glance of Fig. 6, there is a remarkable spin modulation of the electron and ion counts, the maxima of which are detected around zero degree of the sun angle. This spin modulation is, therefore, caused by solar radiation; that is, photoelectrons produced at the inner surface of the sensor and the satellite surface, and also the solar UV radiation itself that impinges onto the channeltrons by random reflection. In this figure, the background counts are found to be negligibly small except around zero degree of the sun angle. The day, November, 6, 1978, was a very quiet day; there occurred no substorm and $\Sigma K_{p}=4^{+}$. Figure 6 shows no significant electron flux above a few $\mathrm{keV}$, though there is a moderate flux of high-energy ions which would be the outer radiation belt ions in such a quiet day. Electrons 


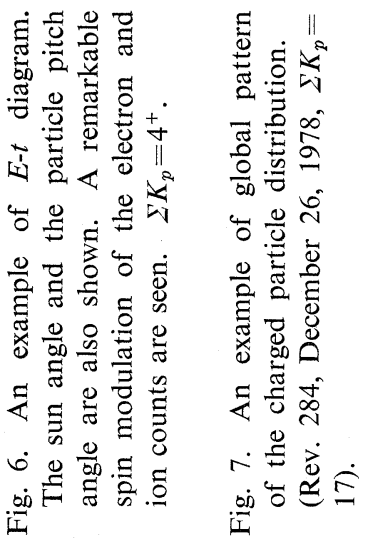

ש̃

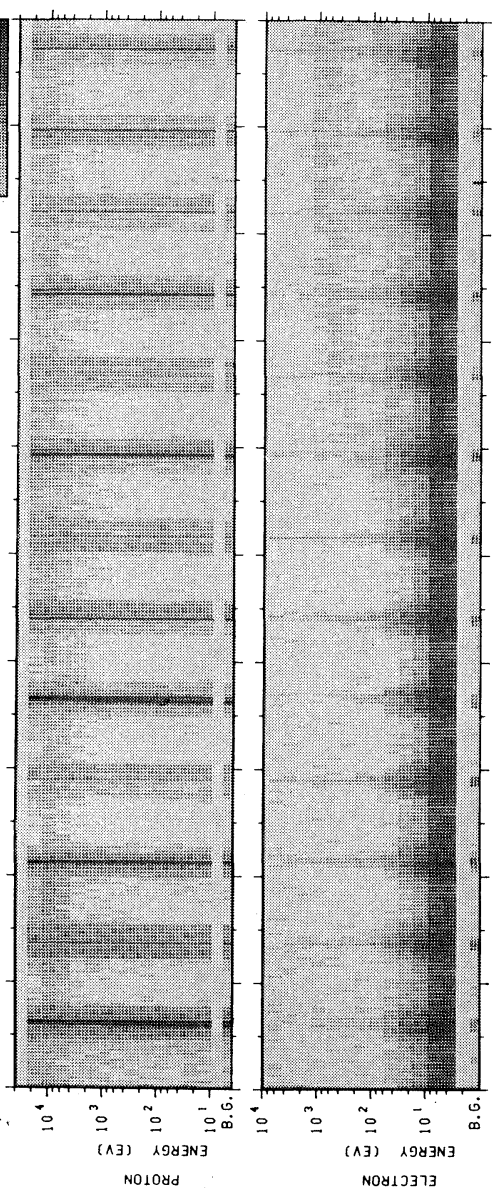

$$
\text { 定 }
$$


with the energy of several hundred $\mathrm{eV}$ to $1 \mathrm{keV}$ appear around $1118 \mathrm{UT}$ ( $L \cong 6.3$, 2137 MLT, $-40.4^{\circ}$ geomagnetic latitude). These electrons show a weak pitch angle anisotropy (which might be obscure in this figure).

Although such data presentation of the 20-min data as Fig. 6 provides us with detailed information of charged particle distribution that can be easily compared with other observations such as VLF emissions, it is also desirable to show a global pattern of the charged particle distribution in one panel. Figure 7 shows an example of global pattern of the charged particle distribution which was reproduced from EXOS-B WAVE/PARTICLE SUMMARY (produced by Drs. A. Nishida and K. Maezawa). In this figure, the respective counts of 13 channels for electrons and ions are shown every $64 \mathrm{sec}$ with gray-shading, the level of which is shown in Table 3. The counts were averaged in 10 frames during $64 \mathrm{sec}$ (64 frames when

Table 3. Grey-shading level of Fig. 7.

\begin{tabular}{|c|c|c|}
\hline Electron & Ion & Shading level \\
\hline 0.3 & $\mathrm{C} \leqq$ & 0 (void) \\
\hline $0.3<\mathrm{C} \leqq$ & $0.1<\mathrm{C} \leqq$ & 1 \\
\hline $1.0<\mathrm{C} \leqq$ & $0.3<\mathrm{C} \leqq$ & 2 \\
\hline $3.0<\mathrm{C} \leqq 10.0$ & $1.0<\mathrm{C} \leqq$ & 3 \\
\hline $10.0<\mathrm{C} \leqq 30.0$ & $3.0<\mathrm{C} \leqq 10.0$ & 4 \\
\hline $30.0<\mathrm{C} \leqq 100.0$ & $10.0<\mathrm{C} \leqq 30.0$ & 5 \\
\hline $100.0<\mathrm{C} \leqq 300.0$ & $30.0<\mathrm{C} \leqq 100.0$ & 6 \\
\hline $300.0<\mathrm{C} \leqq 1000.0$ & $100.0<\mathrm{C} \leqq 300.0$ & 7 \\
\hline $1000.0<\mathrm{C} \leqq 3000.0$ & $300.0<\mathrm{C} \leqq 1000.0$ & 9 \\
\hline $3000.0<\mathrm{C}$ & $1000.0<\mathrm{C}$ & 10 \\
\hline
\end{tabular}

Here, $C$ is the counts/sec and the shading level of No. 8 is not used.

$1024 \mathrm{bits} / \mathrm{sec}$, or 16 frames when $256 \mathrm{bits} / \mathrm{sec}$ ) so as to eliminate the data contaminated by solar radiation. The data shown in Fig. 7 was obtained on Rev. 284 on December 26, 1978. The geomagnetic condition was that there occurred a magnetic storm on the previous day and a substorm occurred at $0730 \mathrm{UT}$ on this day $\left(\Sigma K_{p}=17\right)$. Hot electrons appeared first around $0800 \mathrm{UT}\left(L \cong 5.40,2045 \mathrm{MLT},-17.36^{\circ}\right.$ geomagnetic latitude), but the energy was rather soft (several hundred $\mathrm{eV}$ ). The electron energy and flux were increased with time, and at last, intense (burst-like) $\mathrm{keV}$-electrons were detected for $30 \mathrm{~min}$ around $1030 \mathrm{UT}$ ( $L \cong 5.42,2215 \mathrm{MLT},-5.08^{\circ}$ geomagnetic latitude) when strong VLF emission (like a pure tone) was observed (MATSUмото et al., 1979). Then, the keV-electrons were decreased with time (with decreasing of $L$-value). Significant flux of high-energy ions were detected throughout the orbit. Significant flux of lower-:nergy ions were detected after 1130 UT ( $L \cong 4.76,2252 \mathrm{MLT}, 0.78^{\circ}$ geomagnetic latitude), corresponding to the decrease of the keV-electrons.

Counts of each channel every frame, and average counts and average number flux of each channel every subframe ( 256 frames) are deduced in order to obtain 
$C$ - $t$ diagram and $E$-number flux diagram (energy spectrum). In the averaging procedure, the data contaminated by the solar radiation were discarded in the following way:

1) The time when the counts of $3.8 \mathrm{eV}$-electron (channel 13) show the maximum value is defined as a time when the sensors direct towards the sun and named ST.

2) Ion data in all channels during $\mathrm{ST} \pm 20 \mathrm{sec}$, electron data in channels $1 \sim 9$, i.e., $E_{e} \geqq 33.4 \mathrm{eV}$, during $\mathrm{ST} \pm 10 \mathrm{sec}$, and electron data in channels $10 \sim 13$, i.e., $E_{e}<33.4 \mathrm{eV}$ during $\mathrm{ST} \pm 32 \mathrm{sec}$ are discarded.

Figure 8 shows the variation of average counts of several channels on Rev. 284 ( $C$ - $t$ diagram). The $C$ - $t$ diagram is more convenient to see the variation of counts of specific energies than $E$ - $t$ diagram. The characteristic variation of the particle counts such as the peaks of counts of high energy electrons seen in Fig. 8 was not observed in almost all the other orbits.

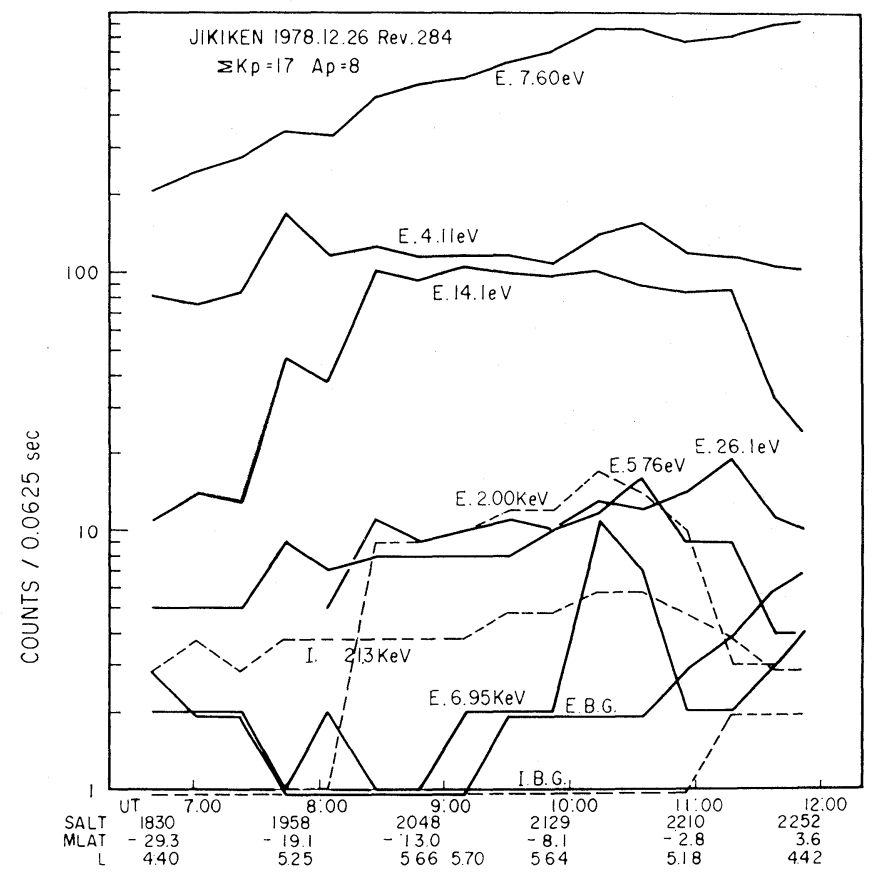

Fig. 8. Variation of the average counts on Rev. 284.

Figures 9 is examples of the $E$-number flux (averaged over one subframe) diagrams, that is, the energy spectra of electrons and ions on Rev. 284. The dashed lines in Figs. 9(a), (b), (c) and (d) are the power law distributions approximated by the least square method using the high energy 7 points. Figure 9(a) shows the 


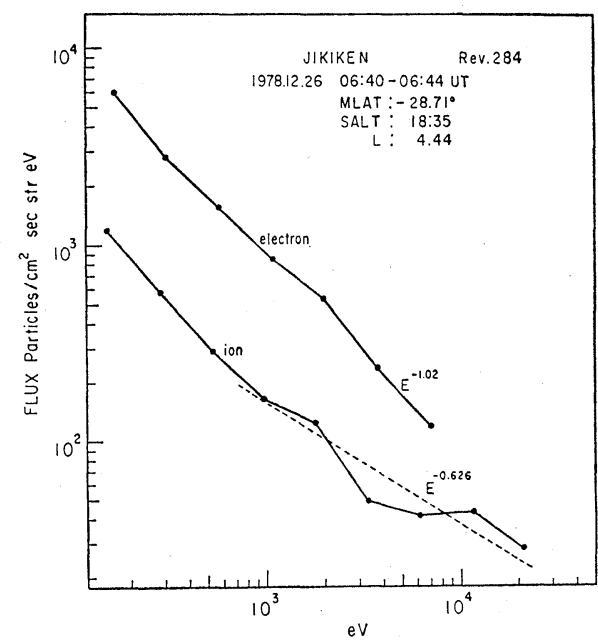

(a)

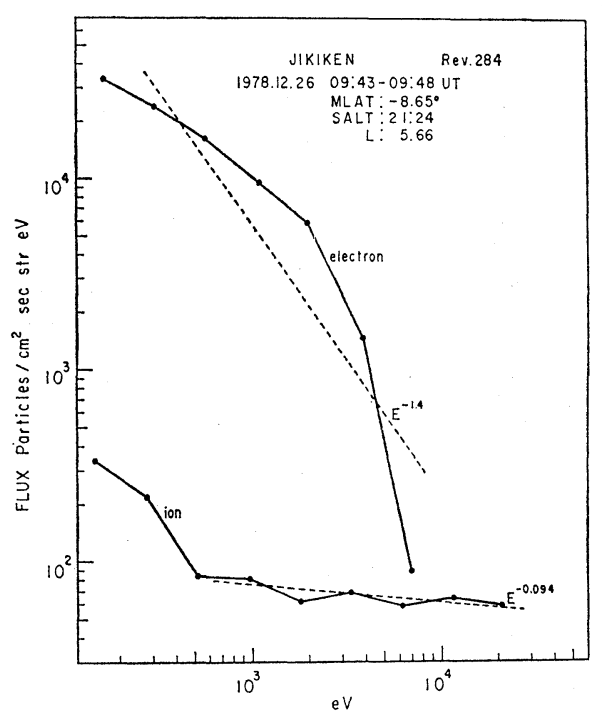

(c)

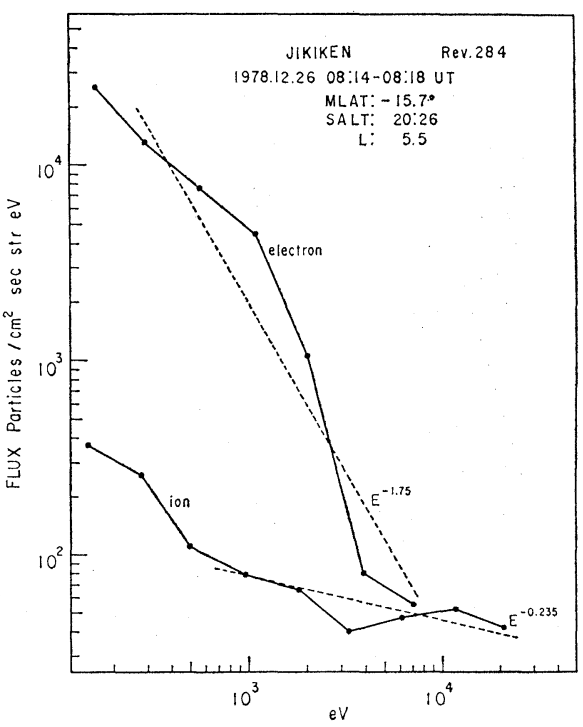

(b)

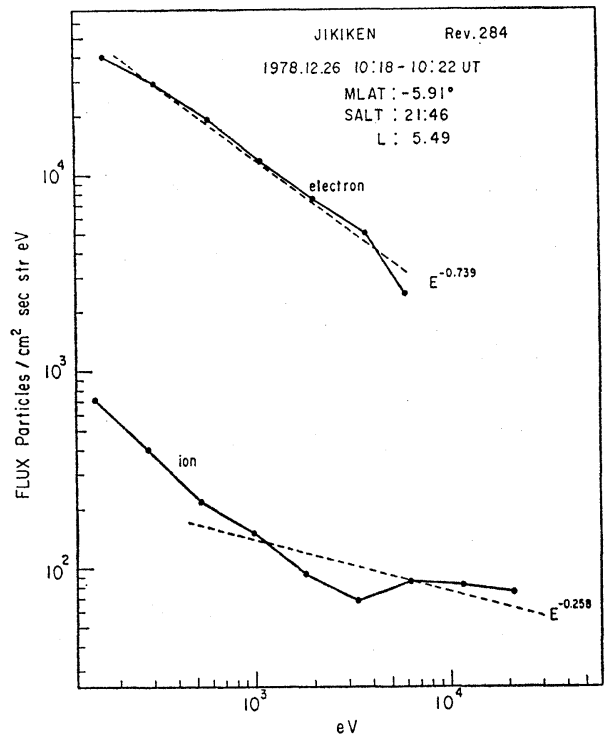

(d)

Fig. 9. Energy spectra of charged particles on Rev. 284 at respective time intervals.

energy spectra before the substorm. Figures 9(b), (c) and (d) are the energy spectra after the substorm, showing that the energy spectrum of electrons become harder with time. When the electron fluxes of several hundred $\mathrm{eV}$ energy increased (Fig. 


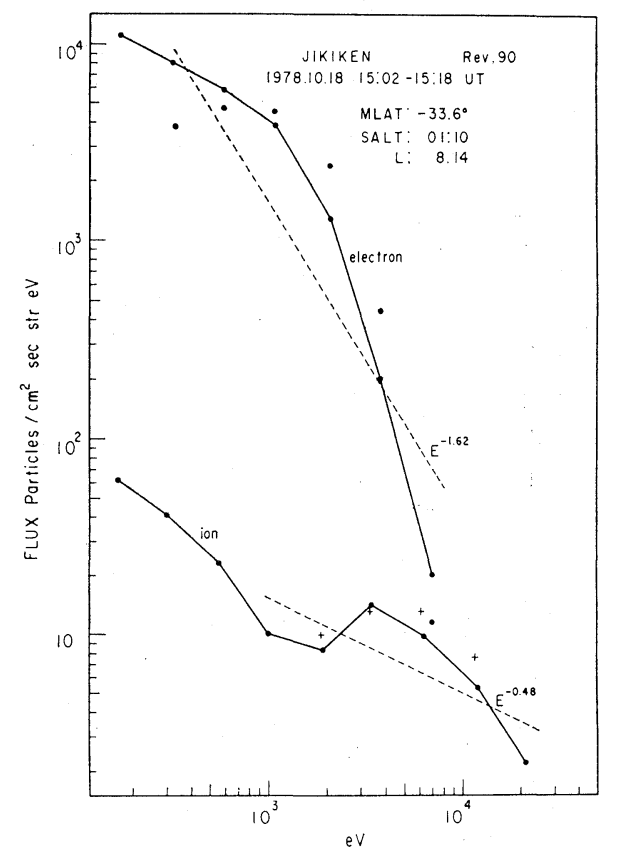

(a)

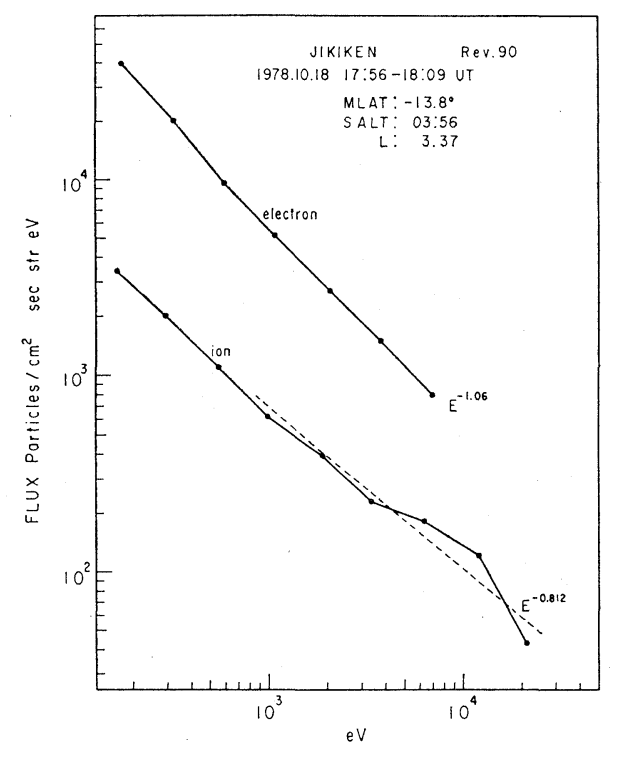

(c)

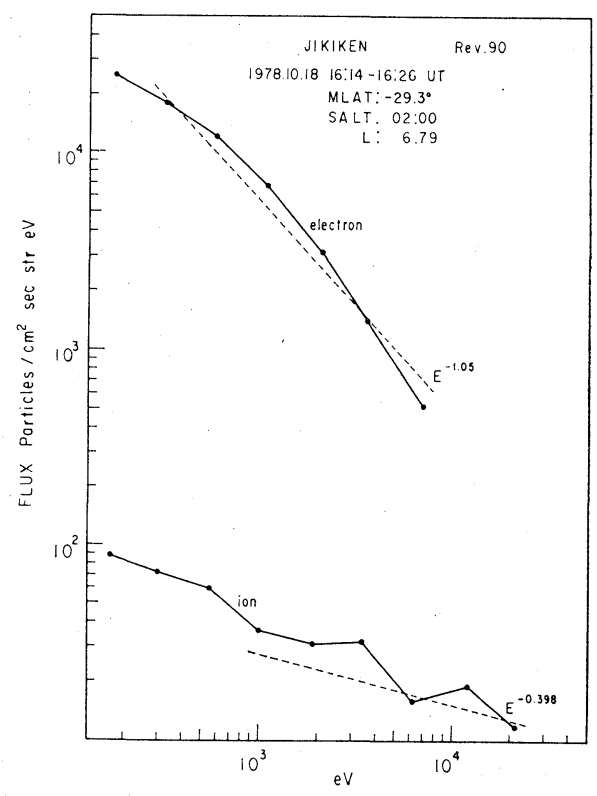

(b)

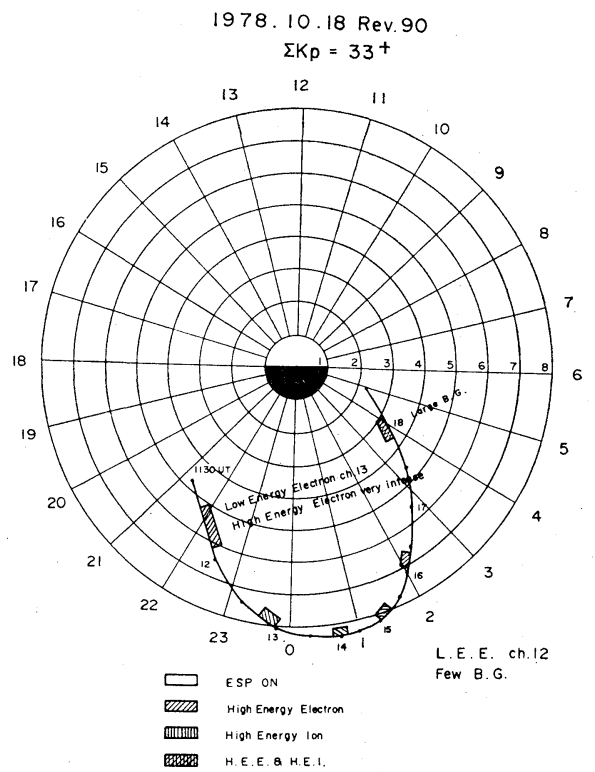

(d)

Fig. 10. (a), (b) (c), Energy spectra of charged particles on Rev. 90 (October 18, 1978, $\Sigma K_{p}=33^{+}$) at respective time intervals. (d) $L$-MLT plot for Rev. 90 . 
9(c)), VLF hiss emission was observed (Матsumoto et al., 1979).

Figures 10(a), (b) and (c) are the energy spectra on Rev. 90 (October 18, 1978). The dashed lines in Figs. 10 are the same as those of Figs. 9, and $\bullet$ (dots) and + (pluses) in Fig. 10(a) are Maxwellian distribution approximated using high energy 6 points. $T_{e}, N_{e}, T_{i}$ and $N_{i}$ are $4.65 \mathrm{keV}, 1.99 \times 10^{-2} / \mathrm{cm}^{3}, 750 \mathrm{eV}$, and $6.94 \times$ $10^{-2} / \mathrm{cm}^{3}$, respectively. Measured fluxes seem to be approximated by the sum of a power law distribution and a Maxwellian distribution.

The ion flux increased by an order of magnitude around 1803 UT (Fig. 10(c)). This is bacause JIKIKEN was at the outer radiation belt, and the peak position of low energy ions moved from $L=5 \sim 8$, the quite time position, to $L=3 \sim 4$ due to the magnetic field disturbance. This is considered to be the ring current ions which are injected from the plasma sheet on the disturbed days after a magnetic storm. The geomagnetic conditions were such that there occurred S.S.C. at 0430 UT of the previous day (October 17) which continued to 1600 UT of the next day (October 19), 5 substorms occurred on this day $(0350,0405,0625$ and 1105 UT), and after 1610 UT magnetic field was disturbed $\left(\Sigma K_{p}=33^{+}\right)$. Figure $10(\mathrm{~d})$ is the $L$-MLT plot for Rev. 90. The $L$-MLT plot is obtained by the following procedure:

1) Plot the satellite orbit onto the $L$-MLT coordinate.

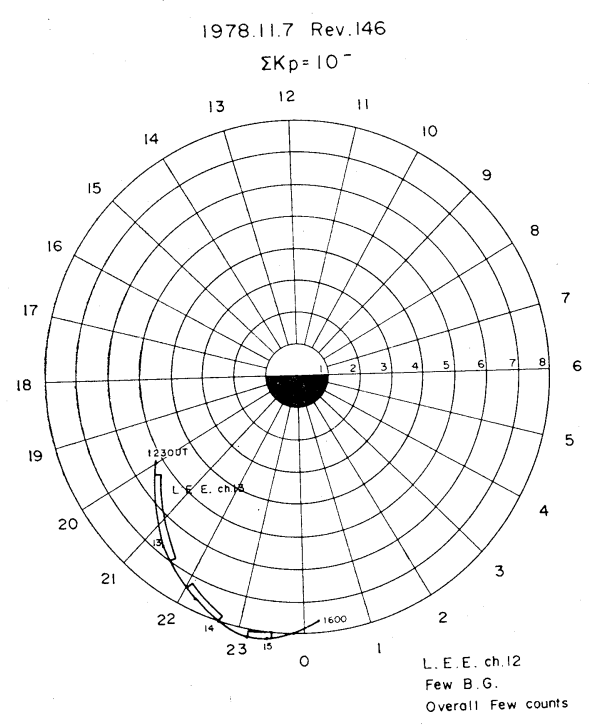

(a)

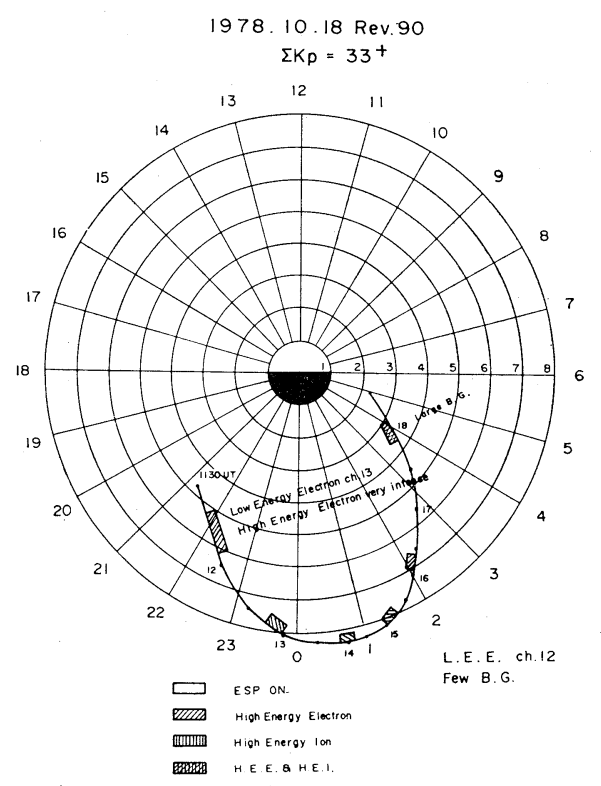

(b)

Fig. 11. (a) An example of the L-MLT plot on quiet days when the satellite orbit is in the midnight sector. (b) An example of the $L$-MLT plot on disturbed days when the satellite orbit is in the midnight sector. Positions where ESP was operating, counts of high energy electrons and ions were large are indicated. 
2) Positions where ESP was operating are indicated on the orbit.

3) Positions where the high energy 4 channels of electrons $\left(E_{e} \geqq 760 \mathrm{eV}\right)$ have more than 5 counts $/ 62.5 \mathrm{msec}$ are indicated as well as positions where the high energy 4 channels of ions $\left(E_{i} \geqq 2.4 \mathrm{keV}\right.$ ) have more than 4 counts $/ 62.5 \mathrm{msec}$ in the case of low level channeltron high voltage, or more than 10 counts $/ 62.5 \mathrm{msec}$ in the case of middle level channeltron high voltage.

4) The channel with largest count in the low energy three channels of electrons, that is, channel $11(13.1 \mathrm{eV})$, channel $12(7.1 \mathrm{eV})$ and channel $13(3.8 \mathrm{eV})$ is indicated.

5) Whether there are large background counts or not is also indicated.

When ESP was operating, high energy electrons were always observed, and around 1800 UT high energy ions were also observed, but this increase perhaps occurred earlier somewhere around 1700 UT because intense HF and VLF emission was observed after this time. To our regret, it was not be able to decide when and where the high energy ions began to increase, because the ESP was not operating in order to save the battery power.

In order to investigate the general features of the charged particle distributions in the inner magnetosphere, $L$-MLT plots are accumulated. These $L$-MLT plots are classified according to $\Sigma K_{p}$ and MLT. Figures 11(a) and 12(a) are the typical

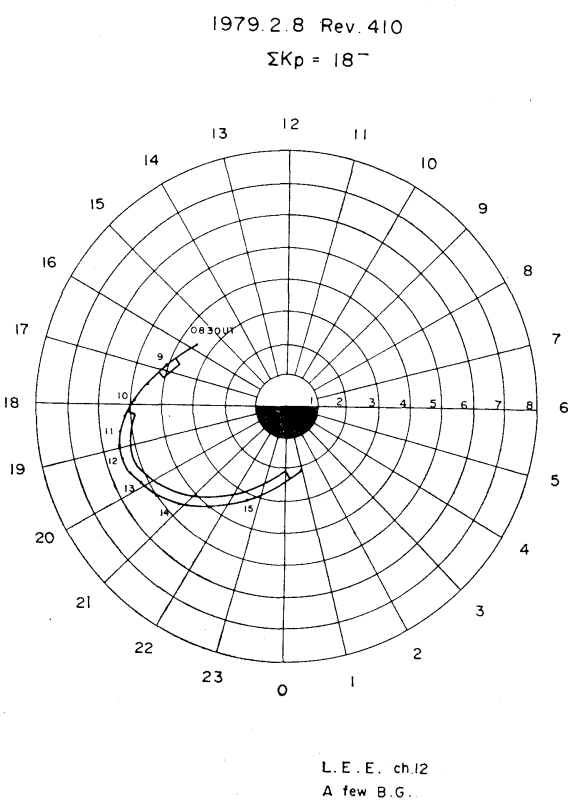

(a)

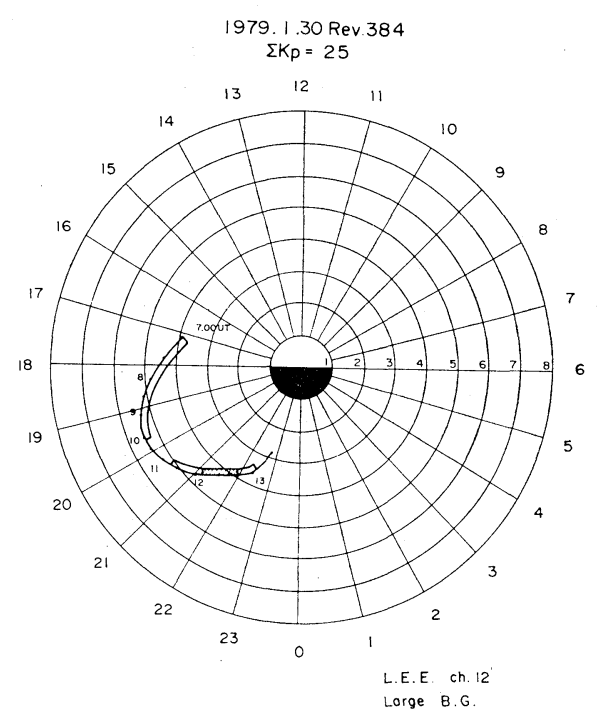

(b)

Fig. 12. (a) An example of the $L$-MLT plot on quiet days when the satellite is in the dusk sector. (b) An example of the L-MLT plot on disturbed days when the satellite is in the dusk sector. 
examples of $L$-MLT plots on quiet days, and Figs. 11(b) and 12(b) are those on disturbed days. The satellite orbit of Figs. 12 differs considerably from that of Figs. 11; that is, the midnight sector in Figs. 11 and the dusk sector in Figs. 12.

Figures 11(a) and 12(a) show that counts of high energy electrons and ions are rare on the quiet days from the midnight to the dusk sector of the magnetosphere. Similar features are seen in the data not shown here. Figures 11(b) and 12(b) show that there are almost always counts of high energy electrons on the disturbed days at $L \gtrsim 4$. These features are the same in the data not shown here.

The following statistical conclusions are deduced from Figs. 11 and 12, and the data not shown here.

1) On quiet days (Rev. 86, Rev. 146, Rev. 276, Rev. 410)

Significant fluxes of electrons and ions in the keV-energy range were rarely observed, since the plasma sheet is far beyond the satellite orbit on such days.

2) On disturbed days (Rev. 90, Rev. 250, Rev. 253, Rev. 273, Rev. 628)

Intense fluxes of $\mathrm{keV}$-electrons were observed at $L \gtrsim 4$, suggesting plasma injection from the plasma sheet. Ring current ions in the energy range above $1 \mathrm{keV}$ were observed after the magnetic storm.

There are some exceptions to the above conclusions.

1) Though December 26, 1978 (Rev. 284) was moderately quiet day, considerable high energy particles were observed (see Figs. 7, 8 and 9), and the $L$-MLT plot is shown in Fig. 13. This is considered due to S.S.C. occurred on the previous

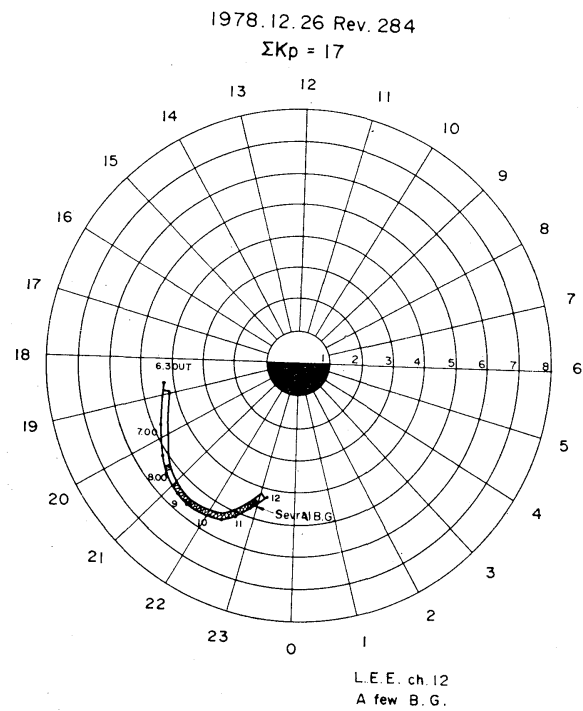

Fig. 13. L-MLT plot for Rev. 284. This is an exceptional case of the $L$-MLT plot on quiet days. 


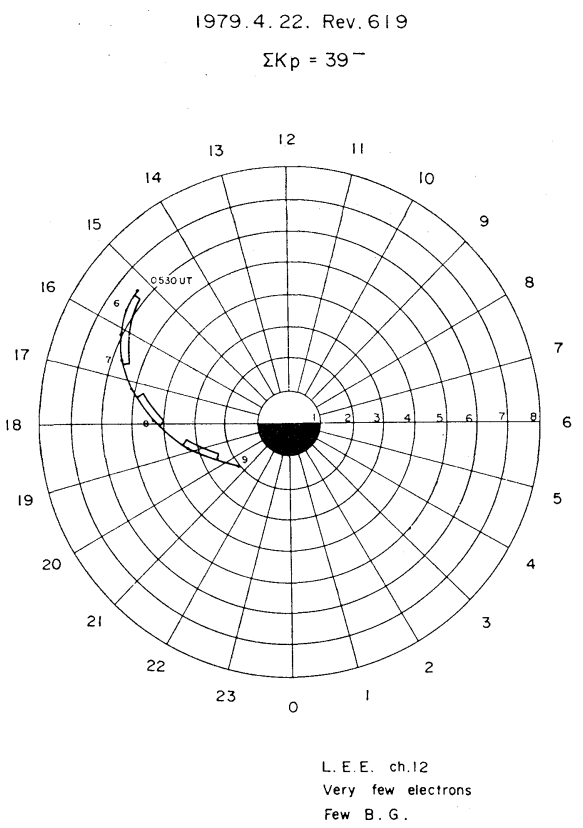

(a)

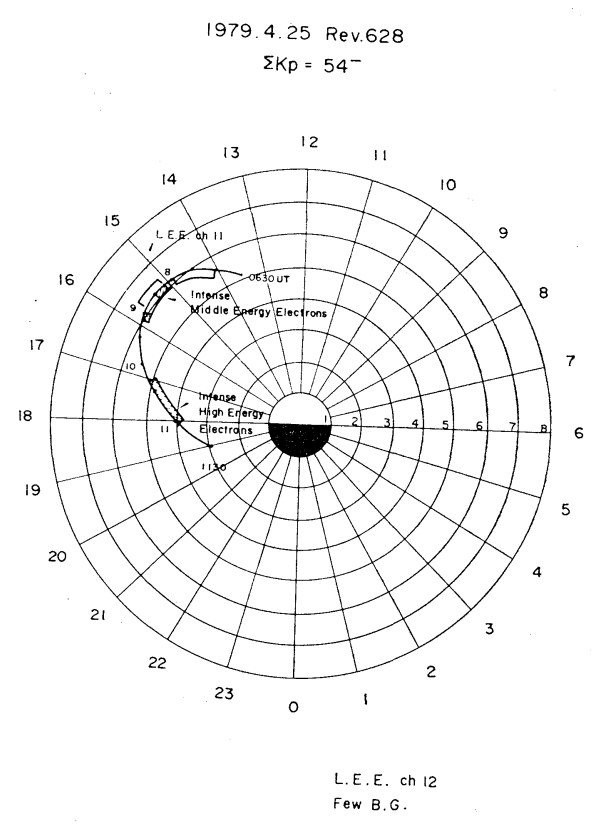

(b)

Fig. 14. (a) $L$-MLT plot for Rev. 619. This is an exceptional case of the $L$-MLT plot on disturbed days. (b) $L$-MLT plot for Rev. 628. Satellite orbits of Figs. 14(a) and 14(b) are similar, but the counts of the high energy particles are very different.

day, but it is also able to consider that the substorm occurred 0730 UT on this day injected the high energy particles from the plasma sheet. However, it is the question whether only the substorm without S.S.C. can inject the charged particles from the plasma sheet or not. It is necessary to investigate the particle distributions after substorms in detail.

2) Though April 22, 1979 (Rev. 619) was the very disturbed day $\left(\Sigma K_{p}=39^{-}\right)$, $\mathrm{keV}$-electron fluxes were not observed. The $L$-MLT plot is shown in Fig. 14(a). If it is due to MLT dependence, it contradicts to the situation of Rev. 628 (April $25,1979)$, the $L$-MLT plot of which is shown in Fig. 14(b). That is, both orbits are in the late afternoon sector. The geomagnetic conditions of the previous day and the day before the previous day may affect the particle distributions. For instance $\Sigma K_{p}$ of the previous day was 25 , and $\Sigma K_{p}$ of the day bebore the previous day was 4. No explanation can be given to this phenomenon now.

In order to clarify this inconsistensy, and in order to make clear the MLT dependence, and $K_{p}$ dependence of charged particle distributions, more data accumulation are necessary. 


\section{Concluding Remarks}

Data processing of ESP on board JIKIKEN has just begun. Summarized results are as follows:

1) On quiet days, significant fluxes of electrons and ions in the keV-energy range are rarely observed since the plasma sheet is far beyond the satellite orbit on such days.

2) On disturbed days intense fluxes of keV-energy particles are observed at $L \gtrsim 4$, suggesting plasma injection from the plasma sheet. The injected keV-electrons at times show good correlation with VLF emission activities, indicating the wave-particle interaction in the magnetosphere.

From now on, to clarify the behavior of the charged particles in the magnetosphere, much more data acquisition is expected. Further, comparison with the wave data, collaboration with the active experiments must be done.

The authors would like to express their sincere thanks to Proffessor T. Obayashi of ISAS, Univ. of Tokyo, and other staff of JIKIKEN team. They are also grateful to Drs. H. Iwakura, S. Saito, S. Miyatake of electro-communication Univ., and K. Hashimoto of Kyoto Univ. for their efforts in constructing and supporting the data acquisition system. They are also grateful to Drs. A. Nishida of ISAS, Univ. of Tokyo and K. Maezawa of Nagoya Univ. for the production of summary plot, and to JIKIKEN tracking team.

\section{REFERENCES}

Matsumoto, H., S. Miyatake, K. Tsuruda, A. Morioka, J. Ohtsu, H. Oya, and I. Kimura, VLF wave observation by EXOS-B (JIKIKEN) satellite, Magnetospheric Study 1979, in Proceeding of the International Workshop on Selected Topics of Magnetospheric physics, pp. 219-223, Japanese IMS Committee, Tokyo, March 1979.

Paschmann, G., E. G. Shelly, C. R. Chappel, R. D. Sharp, and L. F. Smith, Absolute efficiency measurements for channel electron multipliers utilizing a unique electron source, Rev. Sci. Instrum., 41, 1706-1711, 1970. 